\title{
Review
}

\section{Preclinical Models of Encephalopathy of Prematurity}

\author{
Lauren L. Jantzie ${ }^{a, b}$ Shenandoah Robinson ${ }^{c, d}$ \\ Departments of a Pediatrics and ${ }^{\mathrm{b}}$ Neurosciences, University of New Mexico, Albuquerque, N. Mex., and \\ Departments of ${ }^{\mathrm{c}}$ Neurosurgery and ${ }^{\mathrm{d}}$ Neurology, Kirby Center for Neurobiology, Boston Children's Hospital, \\ Harvard Medical School, Boston, Mass., USA
}

\section{Key Words}

Cerebral palsy · Chorioamnionitis · Encephalopathy of prematurity · Hypoxia-ischemia · Inflammation · Lipopolysaccharide · Perinatal brain injury · Preclinical models · Preterm birth · Subplate

\begin{abstract}
Encephalopathy of prematurity (EoP) encompasses the central nervous system (CNS) abnormalities associated with injury from preterm birth. Although rapid progress is being made, limited understanding exists of how cellular and molecular CNS injury from early birth manifests as the myriad of neurological deficits in children who are born preterm. More importantly, this lack of direct insight into the pathogenesis of these deficits hinders both our ability to diagnose those infants who are at risk in real time and could potentially benefit from treatment and our ability to develop more effective interventions. Current barriers to clarifying the pathophysiology, developmental trajectory, injury timing, and evolution include preclinical animal models that only partially recapitulate the molecular, cellular, histological, and functional abnormalities observed in the mature CNS following EoP. Inflammation from hypoxic-ischemic and/or infectious injury induced in utero in lower mammals, or actual prenatal delivery of more phylogenetically advanced mammals, are likely to be the most clinically relevant EOP models, facilitating translation to benefit infants. Injury timing, type, severity, and pathophysiology need to be optimized to address the
\end{abstract}

specific hypothesis being tested. Functional assays of the mature animal following perinatal injury to mimic EoP should ideally test for the array of neurological deficits commonly observed in preterm infants, including gait, seizure threshold and cognitive and behavioral abnormalities. Here, we review the merits of various preclinical models, identify gaps in knowledge that warrant further study and consider challenges that animal researchers may face in embarking on these studies. While no one model system is perfect, insights relevant to the clinical problem can be gained with interpretation of experimental results within the context of inherent limitations of the chosen model system. Collectively, optimal use of multiple models will address a major challenge facing the field today - to identify the type and severity of CNS injury these vulnerable infants suffer in a safe and timely manner, such that emerging neurointerventions can be tailored to specifically address individual reparative needs.

(c) 2015 S. Karger AG, Basel

\section{Introduction}

Encephalopathy of prematurity (EoP) encompasses the central nervous system (CNS) abnormalities that are associated with CNS injury from preterm birth [1]. While white matter injury of periventricular leukomalacia was previously the most obvious finding on neonatal cranial ultrasounds, over time it has become evident that

\section{KARGER 125}

C 2015 S. Karger AG, Basel

$0378-5866 / 15 / 0375-0277 \$ 39.50 / 0$

E-Mail karger@karger.com

www.karger.com/dne
Shenandoah Robinson, MD

Departments of Neurosurgery and Neurology

Boston Children's Hospital, 300 Longwood Ave.

Boston, MA 02115 (USA)

E-Mail Shenandoah.robinson@ childrens.harvard.edu 
perinatal complications associated with preterm birth affect not only the developing cerebral white matter but also the entire CNS $[1,2]$. In the past two decades in regions with advanced neonatal intensive care unit (NICU) care, a decline in white matter injury severity has occurred with a shift from cystic periventricular leukomalacia to the now more common diffuse white matter gliosis. A concomitant shift in improvement in cognitive and behavioral deficits, however, has not been observed [3], which supports the concept that EoP involves more than white matter injury. EoP encompasses white matter injury with loss of immature oligodendrocytes and axons, premature loss of subplate, loss of thalamic neurons, impaired thalamocortical connections, and loss of migrating $\gamma$-aminobutyric acid (GABAergic) neurons [2]. Despite rapid progress in neuroscience, we still have a relatively limited understanding of how the cellular and molecular CNS injury from preterm birth manifests as the myriad of chronic neurological deficits in children who are born preterm. Causes of perinatal brain injury from preterm birth vary in type and severity, and individual variation in the susceptibility to those diverse insults creates additional complexity. Moreover, a wide gulf exists between the patterns of CNS injury observed emerging from advanced NICUs and the general population of preterm infants worldwide. A major challenge facing the field today is identifying the extent of CNS injury these vulnerable infants are suffering on a molecular level in real time, such that infants can be stratified and receive emerging neurointerventions in specifically tailored and indicated regimens rather than a single regimen for all. Given these challenges of repairing the injured developing brain, models used to test specific hypotheses in preclinical studies to advance the care of these children need to be carefully chosen to adequately mimic the human condition of EoP (fig. 1).

\section{EoP: Clinical Considerations}

In 2010, there were approximately 15 million preterm births (gestation $<37$ weeks) worldwide, and direct complications from preterm birth accounted for approximately $35 \%$ of the world's neonatal deaths [4]. Beyond the neonatal mortality, preterm birth extensively alters the trajectory of neurodevelopment and overall health for these children. In the USA, approximately $25 \%$ of cerebral palsy $(\mathrm{CP})$ results from preterm birth. As gestational age (GA) decreases, the risk of CP and related comorbidities increases. Indeed, $14 \%$ of infants born before 27 weeks' GA develop CP compared to $0.2 \%$ of the general population [5]. As former preterm infants become children and young adults, further neurological deficits become manifest. Besides $\mathrm{CP}$ and the related but milder developmental coordination disorder, impairments of learning, cognition, memory, executive function, vision, and hearing, as well as epilepsy and psychiatric disorders, are often diagnosed. The risks of these neurological deficits specifically increase for infants born extremely preterm ( $<28$ weeks' GA), with up to $50 \%$ experiencing cognitive delay and behavioral problems $[3,6]$. Together, these deficits contribute to a lifelong prematurity-related burden of chronic disease and generate significant cumulative individual, familial, social, and economic impact.

EoP has multiple causes [2], and systemic perinatal inflammation from infection and/or hypoxia-ischemia (HI) is likely to act in concert to potentiate CNS damage from preterm birth. The risks of neurological deficits are higher in preterm infants who are also small for gestational age (weight $<10 \%$ of that expected for GA) $[6,7]$ or who have associated perinatal infection [6-9]. Detailed analyses of placentas from extremely preterm infants show inflammation is more commonly associated with spontaneous early birth [10], and chorioamnionitis is now recognized as a major cause of spontaneous preterm delivery [11]. While the impact of isolated prenatal $\mathrm{HI}$ is challenging to document in humans born preterm, placental perfusion defects compound damage from chorioamnionitis [12].

\section{Improving Translational Science and Therapeutic Target Identification in EoP}

To identify and refine targets for new, effective therapeutic interventions for EoP, it is essential to improve our understanding of how insults in utero and in the perinatal period translate to impaired neurodevelopment. EoP is a constellation of abnormalities that includes diffuse white matter damage and oligodendrocyte loss, axonal injury, premature subplate loss, and neuronal injury to the cerebral cortex, thalamus, basal ganglia, cerebellum, and brain stem $[2,13]$. Postmortem samples from human infants with diffuse white matter gliosis show loss of markers of cerebral GABAergic signaling [14] and loss of expression of the neuron-specific potassium chloride cotransporter 2 (KCC2) compared to brains without evidence of white matter injury [15]. Cerebral cortical layer IV KCC2 upregulation and GABAergic subunit maturation is dependent upon sub- 
Fig. 1. Preclinical models of EoP include preterm deliveries in nonhuman primates and in other mammals chorioamnionitis induced by intra-amniotic or intrauterine endotoxin and/or HI mimicking placental underperfusion. These primary approaches to recapitulating CNS injury associated with preterm birth lead to loss of oligodendroglial lineage cells, axonal injury, premature subplate loss, loss of thalamic neurons, and loss of migrating GABAergic neurons - the key components of EoP. Cumulatively, the cellular and molecular abnormalities manifest as imaging abnormalities, motor deficits, propensity to seizures, and impaired cognition and behavior in the mature brain. EoP leads to CP, epilepsy, cognitive deficits, and behavioral abnormalities such as anxiety, attention-deficit disorder and autism-like behaviors.

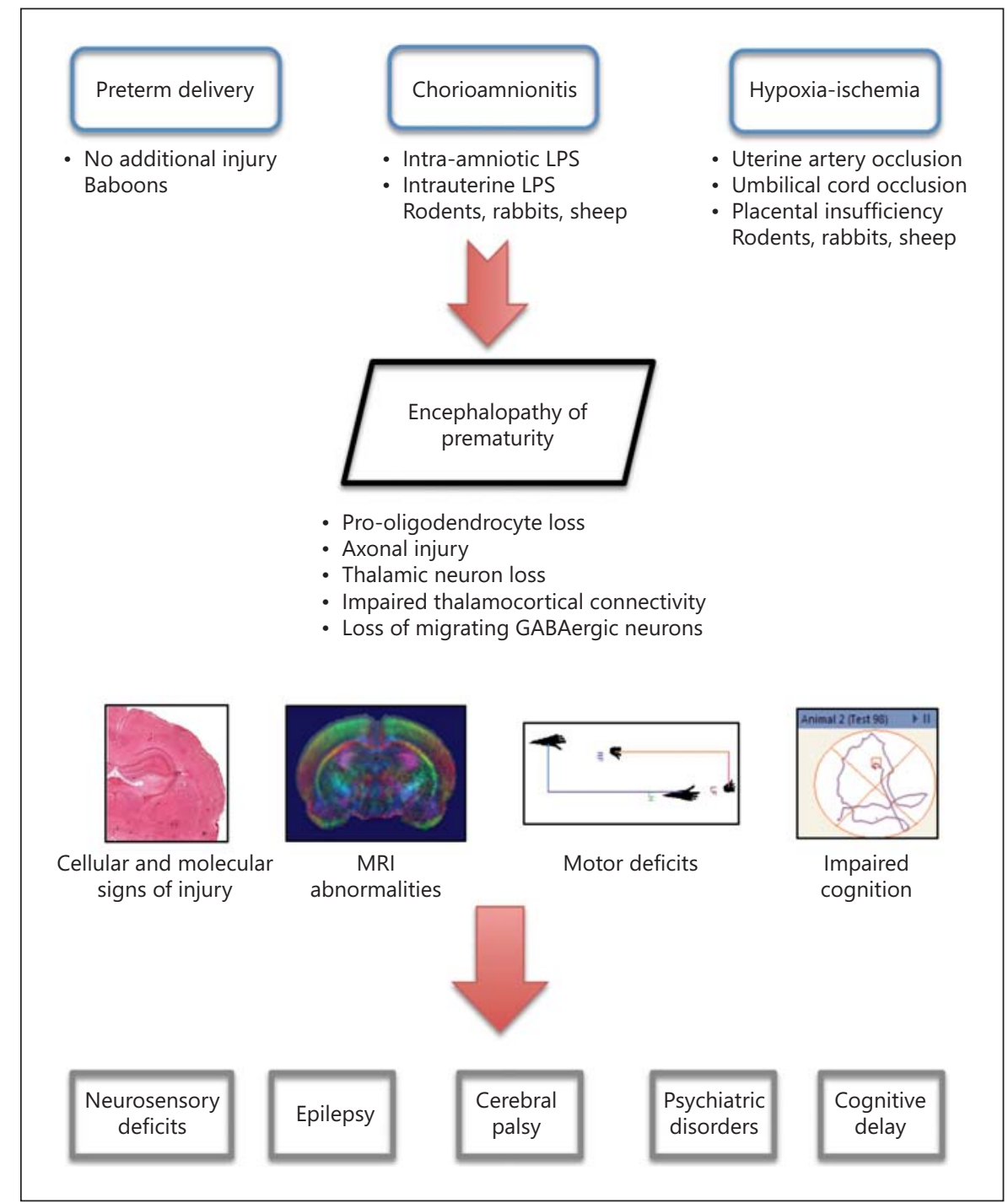

plate neuronal survival [16]. The subplate also contributes to cerebral circuit development and white matter tract refinement $[17,18]$. Excess subplate gliosis [19] and thalamic neuronal loss [20] have been observed in specimens from human preterm infants with white matter injury, and deficient thalamocortical connections were evident with advanced magnetic resonance imaging (MRI) [21]. On a cellular level, EoP affects the development of all neural elements, including neurons, oligodendrocytes, astrocytes, microglia, and the neurovascular unit, as well as related components, including the extracellular matrix, circuit and network formation. The recent demonstration that myelination of cortical pyramidal neuron axons is likely to be involved in cerebral network development [22] reinforces the concept that the integrated development of all neural elements is crucial to cerebral cortical network formation.

Because of the complex interaction of etiologies, neurodevelopmental time course, intricacy of human network formation, overlapping mechanisms, and diverse phenotypes of CNS injury manifest in human preterm infants, EoP is difficult to model well in animals (table 1). As discussed above, EoP is also challenging to model because multiple cell types are injured, intersecting pathophysiological pathways converge and overlap, and the immune response is also undergoing maturation. There are cell-type specific vulnerabilities (immature oligodendrocytes) [23] and diverse developmentally regulated pathways (subplate, antioxidants, transporters, and receptor subunits) [24-28]. Preclinical models of EoP need 
Table 1. Advantages, disadvantages and controversies involved in selecting animal models of EoP

\begin{tabular}{|c|c|}
\hline Species & Advantages \\
\hline Mouse & $\begin{array}{l}\text { Ease of use and relatively low resource utilization } \\
\text { Neural cell development and entire CNS is well } \\
\text { studied with reliable in vitro and in vivo assays } \\
\text { Prenatal manipulation available } \\
\text { Large litters } \\
\text { Extensive molecular and cellular techniques and } \\
\text { reagents available } \\
\text { Validated motor, seizure threshold, behavioral, } \\
\text { and cognitive testing exists } \\
\text { Advanced imaging with MRI readily available } \\
\text { Commonly used to test novel interventions } \\
\text { Genetic approaches available }\end{array}$ \\
\hline
\end{tabular}

Disadvantages

Requires insult induction

(HI, endotoxin)

Small lissencephalic brain

Little white matter and subplate

GABAergic neuronal development

well studied but likely to differ from humans

\section{Controversies}

Postnatal exposure to HI and/or systemic inflammation replicates only postnatal insults without maternal-placental-fetal component

Link between systemic LPS administration to dams and inflammation that is pathogenic in human preterm infants is unclear Commonly used carotid ligation and systemic hypoxia recapitulates focal middle cerebral artery stroke-like pathology, not EoP Neuroimmune development may not replicate human condition well

Rat Ease of use and relatively low resource utilization Requires insult induction Neural cell development and entire CNS is well studied with reliable in vitro and in vivo assays Prenatal manipulation available Large litters (HI, endotoxin) Lissencephalic brain Moderate white matter and subplate

Numerous molecular and cellular techniques and reagents available

Premature loss of subplate exists

Validated motor, seizure threshold, behavioral, and cognitive testing available

Advanced imaging with MRI readily available

MRI abnormalities mimic humans

Commonly used to test novel interventions

Prenatal models mimic human gait, seizure threshold and cognitive deficits of EoP

Rabbit Prenatal manipulation available

Prenatal models mimic human gait deficits

Large litters born

Imaging abnormalities similar to those observed

in human infants

GABAergic neuronal development

well studied but likely to differ

from humans

Postnatal exposure to $\mathrm{HI}$ and/or systemic inflammation replicates only postnatal insults without maternal-placental-fetal component

Link between systemic LPS administration to dams and inflammation that is pathogenic in human preterm infants is unclear Commonly used carotid ligation and systemic hypoxia recapitulates focal middle cerebral artery stroke-like pathology, not EoP Neuroimmune development may not replicate human condition well

Placental unit is a component of prenatal models Advanced imaging with MRI available

MRI abnormalities mimic humans

More complex brain than rodents with greater proportion of white matter

Piglet Gyrencephalic brain

Robust white matter and subplate

Perinatal physiology well characterized

NICU-like environment required for care and piglets can be medically managed similar to human infants Immature organ systems similar to human preterm infants

Susceptible to similar complications as preterm infants, including necrotizing enterocolitis and respiratory distress syndrome Advanced imaging with MRI available MRI abnormalities mimic humans
Requires insult induction

(HI, endotoxin)

Moderate limitations on reagents

Limited study of GABAergic

neuronal development

Tests of cognition and behavioral function and seizure threshold are presently not widely available Involvement of multiple brain regions not fully elucidated

Resource utilization

Extensive neonatal veterinary care required

Moderate limitations on reagents

Limited study of GABAergic

neuronal development

Very limited cognitive, behavioral, seizure threshold, or motor testing available

Advanced imaging with MRI just

emerging
Few survival studies of mature CNS
Rarely used for testing of novel interventions

Rabbit genome is not fully

characterized

Limited potential for genetic manipulation

Brain injury model is not well studied

Limited potential for genetic manipulation 
Table 1. (continued)

\begin{tabular}{|c|c|c|c|}
\hline Species & Advantages & Disadvantages & Controversies \\
\hline Sheep & $\begin{array}{l}\text { Gyrencephalic brain } \\
\text { Robust white matter and subplate } \\
\text { Perinatal physiology well characterized } \\
\text { Can be chronically instrumented } \\
\text { Cerebral blood flow studies possible } \\
\text { EEG readily performed } \\
\text { Short-term pathophysiology well characterized } \\
\text { Advanced imaging with MRI available } \\
\text { MRI abnormalities mimic humans }\end{array}$ & $\begin{array}{l}\text { Resource utilization } \\
\text { Extensive neonatal veterinary care } \\
\text { required } \\
\text { Moderate limitations on reagents } \\
\text { Limited study of GABAergic } \\
\text { neuronal development } \\
\text { Very limited cognitive, behavioral, } \\
\text { seizure threshold, or motor testing } \\
\text { available } \\
\text { Ovine genome is not characterized }\end{array}$ & $\begin{array}{l}\text { Clinical relevance of chronic } \\
\text { instrumentation } \\
\text { Chronic cellular, molecular and } \\
\text { functional abnormalities } \\
\text { undocumented } \\
\text { Studies using endotoxin rarely } \\
\text { involve placental unit } \\
\text { Limited potential for genetic } \\
\text { manipulation }\end{array}$ \\
\hline $\begin{array}{l}\text { Non } \\
\text { human } \\
\text { primates }\end{array}$ & $\begin{array}{l}\text { No additional insult induction required } \\
\text { beyond preterm birth } \\
\text { Multiple brain regions affected } \\
\text { MRI and EEG abnormalities mimic } \\
\text { abnormalities in humans } \\
\text { Complex gyrencephalic brain with white matter } \\
\text { and subplate proportions comparable to humans } \\
\text { Functional assessment possible } \\
\text { Chronic studies performed }\end{array}$ & $\begin{array}{l}\text { Resource utilization } \\
\text { Extensive neonatal veterinary } \\
\text { care required } \\
\text { Moderate limitations on reagents } \\
\text { Moderate study of GABAergic } \\
\text { neuronal development } \\
\text { Extensive complex veterinary } \\
\text { care required }\end{array}$ & $\begin{array}{l}\text { Role of maternal-placental-fetal } \\
\text { unit remains undefined }\end{array}$ \\
\hline
\end{tabular}

to replicate the human condition as much as possible by including a prenatal insult that incorporates the maternal-placental-fetal unit, the heterogeneity of mechanisms of CNS injury observed in the preterm infant and evaluation of both gray and white matter damage and recovery. EoP models should also mimic the pathophysiology at the regional, cellular and molecular levels, including the related development of the neuroimmune system. Thus, to best advance translational objectives, ideal preclinical models of EoP need to do the following: (1) include similar mechanisms of prenatal global injury observed in humans and the interaction of various neural cell types through critical developmental periods, (2) address the multiple components of injury evident throughout the CNS and (3) produce a similar spectrum of functional deficits in the mature animal (fig. 1). No one preclinical model is ideal to test every hypothesis (table 1 ).

Because EoP is difficult to model, it is essential that the experiments be well designed and include proper controls. For example, sham animals should undergo comparable anesthetic and surgical exposure. Especially because of gender differences observed in human clinical studies of outcomes for preterm infants [29], both sexes of animals should be analyzed for functional outcomes, including both sex-specific effects and the effects on both sexes combined. The challenges inherent to EoP that need to be addressed in models include repair of multiple neural cell types, restoration of the microenvironment to support neural cell development, reestablishment of homeostasis, and realignment of multiple developmental trajectories. Similar to the human condition, individual variation in injury exists between animals and to some degree represents an integral component of an accurate preclinical model. Phenotypic variation following early CNS injury in preclinical models is advantageous because individual variation occurs in humans such as spasticity versus dystonia or mixed motor deficits and varying degrees of cognitive and behavioral impairments. In humans and animals, individual variation in severity, recovery, repair, and treatment response can impact the efficacy of novel therapeutics.

\section{Species: Large or Small Mammals}

The choice of animal species used to model EoP impacts the interpretation of experimental data in the context of the inherent limitations posed by the species (table 1). In the simplest terms, birth does not equate to similar points of CNS development across species [30]. Injury to the developing brain from systemic insults and brain injury as a result of early birth are not necessarily the same entity. For example, the rodent CNS at birth, depending on strain (Long-Evans vs. Sprague-Dawley), 
cell type (neurons vs. oligodendrocytes) or pathway or protein being examined (receptor subunit expression), is immature compared to the human CNS at birth, while the human CNS at birth is approximately equivalent to the $\mathrm{P} 10$ rodent.

The timing of injury during gestation has a crucial role in the neurodevelopmental trajectory of the offspring. The spatiotemporal regulation of neural cell developmental stages of proliferation, migration and differentiation differ amongst various mammals [30-32], and these cell-specific developmental programs influence the vulnerability to injury. For example, the overlap of the timing of oligodendrocyte lineage and GABAergic neuronal development with the timing of preterm birth makes these cells particularly susceptible to perinatal insults [14, $33,34]$. In rodents, oligodendrocytes proliferate, migrate and differentiate and myelinate primarily from late gestation through the first 2 postnatal weeks [35], whereas in humans, oligodendrocytes arise during the third trimester and neonatal period and myelination persists over 2-3 years [36]. Oligodendroglial lineage development can be assessed through stage-specific antigens and, although the development of human and animal brains differs in varying degrees of complexity and myelinogenesis, these stage-specific antibodies label similar cells developmentally and biologically across species [23]. The loss of either oligodendrocyte precursor cells or immature oligodendrocytes impacts subsequent myelin formation [37]. With respect to neurons, human GABAergic neurons migrate through the developing white matter and subplate in the third trimester $[14,34]$ and thus are also vulnerable to insults from preterm birth. Thalamic neuronal development is also altered [20], although few mechanistic details regarding thalamic neuronal loss have been elucidated thus far. Therefore, to test a specific hypothesis regarding the developmental impact of an injury, maturation of the neural cell type or pathway should be investigated over a broad developmental window as well as in the mature CNS.

In addition to these cellular and developmental considerations, the type of outcome measures being investigated also impacts the choice of species used because functional maturation also varies amongst mammals [30]. While rodents offer practical advantages with respect to available molecular tools, behavioral assessments and resources, larger mammals have gyrencephalic brains with a more prominent subplate, improved gray-to-white matter ratio and a clinical course of illness more similar to human preterm infants. Studies using genetic approaches and outcome measures such as hippocampal- dependent memory and cognitive and behavioral assessment after injury are more suitable for rodents. By contrast, larger animals can be instrumented and ventilated and have severity of illness and gray-to-white matter ratio more comparable to human preterm neonates, but larger mammals often require full veterinary NICUs and significant resources. Other important considerations include, but are not limited to, the plasticity of CNS or neural cell at the stage of development being investigated, the pace of recovery and injury to other systemic organs that also impact CNS development and repair. Models in sheep can be advantageous to study the brain injury associated with preterm birth [38]. Sheep afford the opportunity to perform clinically relevant studies of white matter, cerebral blood flow, neuroimaging, and electroencephalography (EEG) that are more difficult in smaller mammals. In addition, chronic instrumentation and vascular access also replicate NICU interventions in human infants. However, functional assessment and outcome in the mature ovine brain has been limited, the ovine genome has not been fully characterized, and the molecular tools available to study ovine brain injury are limited. Specifically, it is unclear whether ovine models recapitulate the motor and cognitive deficits commonly diagnosed in former preterm infants and whether cellular and functional damage is sustained in the mature ovine CNS.

Similarly, porcine models may also offer advantages in mimicking the anatomy and physiology of preterm newborns, but preterm porcine models of CNS injury have not yet been fully characterized. Piglet models have been very useful in the study of resuscitation, hemodynamics, $\mathrm{HI}$ encephalopathy, and term brain injury [24,39]. Piglets can also be delivered preterm via cesarean section between 0.79 and $0.98 \mathrm{GA}$, with $0.70 \mathrm{GA}$ being approximately equivalent to a human of 25-27 weeks' GA. Like sheep, very young piglets require extensive neonatal care. Not only are full cesarean sections required, but intact animal NICUs are necessary, as piglets born before 0.87 GA are thermo- and hemodynamically unstable and have immature lungs that require ventilation and surfactant [40]. Preterm pigs born at less than $95 \%$ gestation also have many of the same organ immaturities as the preterm human infant, including increased sensitivity to necrotizing enterocolitis, respiratory distress syndrome and chronic lung disease of prematurity [41]. These similarities accurately recapitulate the needs of preterm human infants, especially in the early neonatal period.

Nonhuman primate models of the brain injury associated with preterm birth have revealed numerous similarities with preterm human infants. Baboons delivered at 
125 days' gestation (0.68 GA) show histological and MRI evidence of white matter loss and ventriculomegaly, concomitant with imaging abnormalities similar to those observed in human preterm infants at term age equivalent, including T2 signal changes, elevated apparent diffusion coefficient, lower relative fractional anisotropy, and higher radial diffusivity values [42]. Importantly, the preterm baboon model differs from other existing animal models in that there is no direct insult applied to the developing brain other than that associated with standard neonatal intensive care and ventilatory support [43]. Thus, the baboon model is unlike the rodent and other mammal models that subject the developing animals to additional insults such as placental insufficiency-induced uterine artery occlusion or inflammation induced by an endotoxin. The combination of having CNS injury evident without an additional insult plus the complex gyral formation of a higher-order species makes this model compelling [43], especially as diffuse white matter injury without cystic lesions is the most common finding, with hippocampal and gray matter cell loss and EEG and MRI abnormalities. Thus, all of the mammals offer prenatal models of injury and advanced imaging with MRI (table 1). Moving forward, a reasonable strategy would be to validate injury mechanism and therapeutic interventions in multiple animal models. Rodent models are ideal to provide for rapid, cost-effective access to cellular and molecular mechanisms and target validation, which could then be subsequently confirmed, as necessary, in large animal models such as the instrumented sheep, preterm piglet or nonhuman primate.

\section{Cross-Talk within the Maternal-Placental-Fetal Unit}

Infants who are born preterm are at risk for a combination of $\mathrm{HI}$ and/or infectious inflammatory insults throughout the perinatal period, and both infection and $\mathrm{HI}$ induce inflammatory signaling, both systemically and in the CNS. Many existing animal models can be categorized according to the location of the injury in relation to the maternal-placental-fetal unit. Models in rats, mice, rabbits, sheep, pigs, and nonhuman primates have contributed information from each level of the maternal-placental-fetal system. However, historically, and despite recent advances in understanding the pathophysiology of EoP, injury models in postnatal rodents have been used, even though in utero inflammation or placental underperfusion is not recapitulated. Many, but not all, postnatal rodent models demonstrate focal stroke-like patho- physiology accompanied by cystic lesions in white and gray matter - or severe cell death in the middle cerebral artery vascular territory - in contrast to the diffuse injury and gliosis observed throughout the brain that is commonly observed in preterm infants in current advanced NICUs. However, models of in utero inflammation from inactivated infectious agents and/or placental $\mathrm{HI}$ that show cellular, histological and functional deficits similar to those observed in humans exist in rodents [35, 44-46], rabbits $[47,48]$ and sheep $[38,49]$. These models mimic the complex maternal-placental-fetal insults that occur in human infants born preterm, which is crucial because chorioamnionitis, placental and amniotic membrane inflammation and placental underperfusion comprise such a large component of human preterm pathophysiology that leads to EoP. Despite these improvements, it is difficult to have preclinical models that are fully representative of every component of EoP. Structurally, rabbits, rats, mice, and higher-order primates like humans have hemochorial placentation with discoid placentas. Sheep, on the other hand, have cotyledonary and epitheliochorial placentas that differ significantly in structure, blood flow, maternal-placental interface, and development. Additionally, actually recapitulating the common clinical scenario in humans of ascending bacterial infections that weaken the amnion and precipitate premature rupture of membranes and/or placental perfusion defects that stress the placental interface and disrupt placental homeostasis is challenging. However, strides have been made to involve multiple components of the maternal-placental-fetal unit and involve in utero inflammation to various degrees. The type of bacterial or viral infection, along with timing during gestation, may impact the neurodevelopmental consequences. As noted above, similar stimuli may induce different CNS phenotypes depending on the timing, severity and repetition of injury administration, and different stimuli may induce very similar CNS phenotypes. This complexity in preclinical models is similar to the wide array of deficits observed in humans who are born very preterm and suffer from a myriad of perinatal insults.

In animals, numerous strategies have been employed to induce inflammation from infectious agents or HI. At the maternal level, inactivated infectious inflammation has been produced with systemic Gram-negative bacterial endotoxin lipopolysaccharide (LPS) administration via intraperitoneal injections, application directly to the cervix, intrauterine injection, or intrauterine infusion either alone $[47,50]$ or in combination with HI [51]. As LPS does not cross the placenta, intraperitoneal injec- 
tions in dams are less likely to produce the same fetal inflammatory response and fetal membrane and vessel involvement as the various forms of intrauterine administration. LPS has the advantage over direct infection with typical intrauterine bacteria in that it activates inflammatory signaling through toll-like receptor 4 without causing active bacterial infection and the associated risk of pathogen spread. Similar to studies using LPS as a Gram-negative bacterial analog to induce inflammatory signaling, laboratories have also investigated maternal inflammation with intraperitoneal injections of inactivated group B streptococcus, which causes placental and neuropathological abnormalities and autistic-like behavior in rats [52]. Similarly, Ureaplasma lipoprotein multiple-banded antigen can simulate Ureaplasma species infection - the most common cause of human chorioamnionitis [53] - but preclinical studies of CNS injury using this agent have not yet been performed. As more inactivated infectious agents become available, it will be informative to determine how they differentially impact neurodevelopment and the efficacy of neuroreparative interventions.

In rodents, steps have been taken to make improvements in the delivery of an inflammatory stimulus in the intrauterine environment. We recently developed an in utero rat model of intra-amniotic LPS administration with or without $\mathrm{HI}$ that induces molecular, histological and functional abnormalities in mature animals, including a spasticity-like motor phenotype that includes toewalking, ataxia, shorter stride length, gait variability, and inconsistency [54]. Although gait abnormalities are present in mature rats, more prominent early postnatal increases in muscle tone are observed in rabbits exposed to prenatal LPS [47], illustrating differences amongst preclinical models. Although prenatal $\mathrm{HI}$ alone in rats produces motor phenotype with cognitive deficits [44] and a lower seizure threshold [55], the addition of LPS-induced inflammation encompasses a component of in utero injury commonly found in extremely preterm births $[10,11]$. Thus, an inactivated infectious stimulus in the uterus can promote processes that lead to fetal CNS damage [8]. Specifically, multiple pathogens can initiate the intrauterine inflammatory process and support the hypothesis that microorganisms isolated from the uterus increase the risk of white matter damage in preterm infants [8]. Further study is necessary to clarify the degree of placental involvement across species and the relationship between the injury observed, placentation, inherent placental structure, and vascular supply to the fetus.

\section{Precipitating Insult: Inflammation from Infectious Stimuli and/or HI}

The induction, evolution and resolution of inflammation in the developing CNS are exceedingly complex and multifactorial. The preterm newborn is capable of intermittent or sustained systemic inflammation that contributes to adverse neurodevelopmental outcomes [56]. Specifically, prolonged, poorly regulated neuroinflammation and inadequate repair are major factors that contribute to chronic deficits [56]. Inflammation in the perinatal brain may persist because inflammation fails to resolve, developmental regulation is insufficient, positive feedback loops between innate and adaptive immune systems are immature, and/or epigenetic mechanisms exist [56]. Notably, preterm newborns are deficient in selected serum anti-inflammatory proteins [57] and have neutrophils resistant to apoptosis that persist and propagate inflammatory signals [58]. The abnormal immune reaction may persist. Monocytes from former preterm children with CP show a heightened response to an LPS challenge compared to monocytes from their preterm peers who have no neurological deficits [59]. Along with limited anti-inflammatory responsiveness, this capacity to sustain inflammation places preterm infants at an increased risk for CNS damage during perinatal development.

In both large and small animal models, the role of inflammation from inactivated infectious agents alone, or in combination with HI, has been controversial. The difficulty arises largely because multiple variables related to experimental design, such as preconditioning and sensitization, alter the observed outcomes [60]. Experimental design factors include the species and age of animals used, type and severity of precipitating insult (including dose, route of administration and dosing regimen) and experimental end points. These various factors make direct study-to-study comparisons challenging [51]. The experimental end points chosen are important because acute and chronic changes differentially affect outcome with respect to myelination, neural cell survival, cellular inflammatory response, and motor function [54]. Undoubtedly, the temporal relationship between LPS and HI insults can be critical $[60,61]$. Similarly, differences in dosage can elicit divergent results. Low concentrations of LPS have been reported to reduce damage prior to $\mathrm{HI}$, whereas higher doses $(300 \mu \mathrm{g} / \mathrm{kg})$ have been reported to potentiate HI damage [62]. The reason for this variability may be related to cellular inflammatory responses and individual changes in microglial activation and reactivity. None of the abovementioned models in rodents are clinically relevant to 
EoP, however, as they occur in postnatal rodents and involve carotid ligation plus systemic hypoxia that results in focal stroke-like pathology, which is uncommon in human preterm infants. In addition, none of these models recapitulate the complexities of the maternal-placental-fetal abnormalities observed in human infants. These models may be useful, however, in addressing important questions regarding systemic inflammation and the molecular mechanisms of cellular sensitization and preconditioning in the immature neuroimmune environment.

Although sheep have a different type of maternal-placental-fetal unit than humans, they do allow more precise studies of physiological manipulation than rodents. In preterm fetal sheep a regimen of acute-on-chronic inflammation is associated with white matter injury and a fetal inflammatory response [63]. By contrast, in fetal sheep at 0.7 GA (broadly equivalent to 28-32 weeks of human development), chronic LPS infusion prior to a period of asphyxia resulted in reduced white matter injury compared to asphyxia alone [63]. Together, these data emphasize the importance of the LPS dosing regimen, time course of injury and route of administration. To facilitate clinical translation, it may be most beneficial to use models that incorporate the placental unit and inflammation from either $\mathrm{HI}$ and/or infectious stimuli to better resolve the contribution of chorioamnionitis to brain injury in human preterm birth. A key characteristic of human EoP is that the developing CNS appears to experience sustained and multiple waves of inflammation due to cumulative insults [56, 64]. Animal models of intrauterine inflammation from inactivated infectious agents reveal insight into the molecular mechanisms underlying fetal CNS injury and may be especially relevant to the clinical scenario. Chronic intra-amniotic administration of LPS to fetal sheep induces microglial activation and subcortical white matter injury, with hippocampal and white matter injury occurring independently of gross T2 volumetric MRI changes [49]. Interestingly, a single intra-amniotic bolus of LPS in the prenatal ovine also leads to microglial activation, astrocyte proliferation and increased apoptosis, which are associated with functional EEG changes [65]. Thus, both isolated and repetitive LPS dosages in utero can induce a sustained pattern of CNS inflammation and injury that mimics the damage observed in human preterm infants.

Similarly, the amount of $\mathrm{HI}$ injury can also be varied. In sheep, Duncan et al. [66] and Rees et al. [67] have investigated $\mathrm{HI}$ in numerous contexts and paradigms, including acute and chronic placental insufficiency. Similar to the gradation of injury found with prenatal transient systemic $\mathrm{HI}$ in rats [35], a relatively prolonged interval of
25 min of umbilical cord occlusion at 0.7 gestation produces a more severe, global HI injury with more robust microglial activation and proliferation, together with white matter injury, EEG suppression and neutrophil influx to the brain [68]. Likewise, we found that increasing intervals of transient systemic HI (TSHI) on E18 in pregnant Sprague-Dawley rat dams induces a graded placental underperfusion defect associated with increasing CNS damage [35]. This timing recapitulates the intrauterine global prenatal insult that occurs in human infants prior to extremely preterm birth at 23-25 weeks' gestation. Previously, we showed that this injury consistently results in white matter astrogliosis, oligodendrocyte loss and axonal disruption in white matter in the cortex similar to alterations observed in human postmortem samples [35]. In this model following injury, $\mathrm{O} 4$-immunoreactive immature oligodendrocytes are most affected and their loss correlates with decreased survival and maturation [69], with the most notable reductions in $\mathrm{O} 4+$ and $\mathrm{O} 1+$ stages of the lineage [35], consistent with previous reports by other investigators [70]. This model also demonstrates premature loss of the subplate, reduced KCC2 expression, a lower seizure threshold, and impaired gait $[25,26$, 55]. Detailed analyses of other components of EoP, including loss of thalamic and GABAergic neurons and the contribution of placental pathology, is underway. The sustained period of perinatal inflammation and gradation of injury observed in humans, as well as in both small and large mammals from various HI and/or LPS insult regimens, supports the need to identify accurate biomarkers to quantify the extent of ongoing CNS injury in real time in both preclinical models of EoP and preterm infants.

To better mimic the high incidence of chorioamnionitis in spontaneous very early preterm birth $[10,11]$ and the related impact of infectious stimuli on CNS development, in utero inflammation from intra-amniotic LPS was added to HI during the E18 laparotomy [54]. Acute postnatal ventriculomegaly and subacute gliosis and white matter loss was observed in pups subjected to dual TSHI + LPS and TSHI alone in the first 2 postnatal weeks [54]. Interestingly, sustained myelin basic protein loss and axonal damage was more prominent in P28 juvenile rats from prenatal TSHI alone compared to animals with prenatal TSHI + LPS. Sophisticated, computerized treadmill gait analyses revealed significant motor impairment with increased ataxia, decreased paw area consistent with toe-walking, reduced stride length, and increased step-to-step variability in all P28 animals subjected to LPS alone, TSHI alone or TSHI + LPS. Limitations of this model include potential variation in the extent of $\mathrm{HI}$ and reperfusion injury from 
transient uterine artery occlusion, although all fetuses become evenly dusky after $60 \mathrm{~min}$ of TSHI. Also, while no effect was noted with sterile intra-amniotic saline injection used as a control for the LPS, loss of amniotic fluid at the time of LPS injection could potentially occur. Although much more work remains, the prenatal TSHI and TSHI + LPS models meet several of the criteria of an EoP model, including causing loss of neurons, oligodendrocytes and axons, loss of subplate and functional deficits in adults that mimic those observed in children born extremely preterm.

Preclinical models with prenatal injury in rabbits have also reported gait abnormalities, with significant spasticity and abnormalities in tone $[47,48]$. Similar to the rat models of prenatal injury $[35,44,46,54]$, the rabbit model is advantageous because it is a fetal model, involves global CNS injury, spares the dam significant effects, and yields large litter sizes via normal delivery [48]. Rabbits develop and retain motor deficits over the first 3 postnatal weeks. Reduced fractional anisotropy and microstructural abnormalities are also observed with advanced MRI techniques $[71,72]$. Although these rabbit models induce similar motor deficits to the rodent models, testing of cognition and behavior (the most burdensome deficits for children who are born preterm) remains challenging in rabbits. Similarly, the contribution of loss of subplate, thalamic and GABAergic neurons following late gestation injury has yet to be investigated well in rabbits or larger mammals. In considering similarities between inflammation induced by $\mathrm{HI}$ and infectious insults leading to gait deficits, it is possible that mechanistic pathways diverge in some respects and converge on others [54, 73]. For example, it is possible that $\mathrm{HI}$ produces a primary injury to oligodendrocytes and neurons followed by secondary microglial activation, whereas LPS may activate microglia as the primary event followed by secondary injury to surrounding neural cells and the neurovascular niche [47].

Dissecting how the specific injuries, alone or cumulatively, impact the multiple components of injury in EoP is essential to refining the targets for intervention. In the future, investigations using preclinical models could lead to substantive mechanistic advances in pathophysiology and guide therapeutic strategies with stratification of preterm infant populations.

\section{Conclusion}

Our limited understanding of the mechanisms underlying in utero insults that adversely affect neurodevelopment following extremely preterm birth hinders the ra- tional design and use of neonatal interventions to mitigate early CNS injury. Human studies of preterm birth reveal a strong correlation between intrauterine and postnatal inflammation, primarily postnatal $\mathrm{HI}$ and subsequent impaired CNS development. Human studies also suggest that different types of injury, alone or in combination, impact the developing CNS via unique mechanisms, and thus preterm infants will most likely require tailored neonatal cocktails of emerging neuroreparative interventions. Given the immense complexity of the human developing CNS and the myriad of insults that affect the maternal-placental-fetal unit, there is no perfect animal model of EoP. However, significant strides have been made over recent years to understand its pathogenesis. While some models may be more or less relevant to the clinical scenario of EoP, each study has advanced the field and improved our understanding of the pathophysiology and potentially viable and safe therapeutic targets such as erythropoietin, $\mathrm{N}$-acetylcysteine and melatonin [74]. As one looks forward, an optimal paradigm will include both rodents and larger animals and subsequent target validation in humans. Preclinical studies provide an essential foundation for carefully conducted clinical studies [38]. Accordingly, progress relies on consistency and selection of the most appropriate model or experimental paradigm for the hypothesis at hand. Given the potential for overlapping mechanisms of infection and HI-related brain injury, placental inflammation and ischemic and inflammatory tolerance are important considerations. Crosstalk and convergent points in the pathophysiology such as toll-like receptors, cytokines and inflammatory cell activation are likely to exist, as do mechanisms for divergence such as erythropoietin receptor expression, repair inflammatory phenotypes and cerebral blood flow autoregulation. Thus, evaluation of the CNS microenvironment and integrated neural components is crucial, as an effective therapeutic strategy or cocktail of interventions for EoP is likely to hinge on the improvement of all neural cells in order to normalize function and repair deficits that prevent children with brain injury associated with prematurity from leading healthy, productive and independent adult lives.

\section{Acknowledgments}

This study received funding from the following sources: NIHNINDS R01 NS060765 (S.R.), Boston Children's Hospital Translational Research Program (S.R. and L.J.), University of New Mexico P30 CoBRE Pilot Program (L.J.), and University of New Mexico Child Health Signature Program (L.J.). 


\section{References}

1 Volpe J: Encephalopathy of prematurity includes neuronal abnormalities. Pediatrics 2005;116:221-225.

2 Volpe J: Brain injury in premature infants: a complex amalgam of destructive and developmental disturbances. Lancet Neurol 2009; $8: 110-124$.

-3 Anderson PJ: Neuropsychological outcomes of children born very preterm. Semin Fetal Neonatal Med 2014;19:90-96.

-4 Blencowe H, Cousens S, Chou D, Oestergaard M, Say L, Moller AB, Kinney M, Lawn J; Born Too Soon Preterm Birth Action Group: Born too soon: the global epidemiology of 15 million preterm births. Reprod Health 2013; 10(suppl 1):S2

5 Maitre NL, Slaughter JC, Aschner JL: Early prediction of cerebral palsy after neonatal intensive care using motor development trajectories in infancy. Early Hum Dev 2013;89: 781-786.

-6 Orchinik LJ, Taylor HG, Espy KA, Minich N, Klein N, Sheffield T, Hack M: Cognitive outcomes for extremely preterm/extremely low birth weight children in kindergarten. J Int Neuropsychol Soc 2011;17:1067-1079.

-7 Leviton A, Fichorova RN, O'Shea TM, Kuban K, Paneth N, Dammann O, Allred EN; ELGAN Study Investigators: Two-hit model of brain damage in the very preterm newborn: small for gestational age and postnatal systemic inflammation. Pediatr Res 2013;73:362-370.

$\checkmark 8$ Leviton A, Allred EN, Kuban KC, Hecht JL, Onderdonk AB, O'Shea TM, Paneth N: Microbiologic and histologic characteristics of the extremely preterm infant's placenta predict white matter damage and later cerebral palsy. The ELGAN study. Pediatr Res 2010; 67:95-101.

-9 O'Shea TM, Shah B, Allred EN, Fichorova RN, Kuban KC, Dammann O, Leviton A; ELGAN Study Investigators: Inflammationinitiating illnesses, inflammation-related proteins, and cognitive impairment in extremely preterm infants. Brain Behav Immun 2013; 29:104-112.

-10 Trivedi S, Joachim M, McElrath T, Kliman HJ, Allred EN, Fichorova RN, Onderdonk A, Heitor F, Chaychi L, Leviton A, Majzoub JA; Extremely Low Gestational Age Newborns (ELGAN) Study Investigators: Fetal-placental inflammation, but not adrenal activation, is associated with extreme preterm delivery. Am J Obstet Gynecol 2012;206:236.e1-e8.

11 Pappas A, Kendrick DE, Shankaran S, Stoll BJ, Bell EF, Laptook AR, Walsh MC, Das A, Hale EC, Newman NS, Higgins RD: Chorioamnionitis and early childhood outcomes among extremely low-gestational-age neonates. JAMA Pediatr 2014;168:137-147.

12 Kaukola T, Herva R, Perhomaa M, Paakko E, Kingsmore S, Vainionpaa L, Hallman M: Population cohort associating chorioamnionitis, cord inflammatory cytokines and neurologic outcome in very preterm, extremely low birth weight infants. Pediatr Res 2006;59: 478-483.

13 Robinson S: Systemic prenatal insults disrupt telencephalon development: implications for treatment. Epilepsy Behav 2005;7:345-363.

14 Robinson S, Li Q, DeChant A, Cohen M: Neonatal loss of $\gamma$-aminobutyric acid pathway expression after human perinatal brain injury. J Neurosurg 2006;104:396-408.

15 Robinson S, Mikolaenko I, Thompson I, Cohen M, Goyal M: Loss of cation-chloride cotransporter expression in preterm infants with white matter lesions: implications for the pathogenesis of epilepsy. J Neuropathol Exp Neurol 2010;69:565-572.

16 Kanold P, Shatz C: Subplate neurons regulate maturation of cortical inhibition and outcome of ocular dominance plasticity. Neuron 2006;51:627-638

17 Kostovic I, Judas M: The development of the subplate and thalamocortical connections in the human foetal brain. Acta Paediatr 2010; 99:1119-1127.

18 Kanold PO, Luhmann HJ: The subplate and early cortical circuits. Annu Rev Neurosci 2010;33:23-48.

19 Pogledic I, Kostovic I, Fallet-Bianco C, AdleBiassette $\mathrm{H}$, Gressen P, Verney C: Involvement of the subplate zone in preterm infants with periventricular white matter injury. Brain Pathol 2014;24:128-141.

-20 Ligam P, Haynes RL, Folkerth RD, Liu L, Yang M, Volpe JJ, Kinney HC: Thalamic damage in periventricular leukomalacia: novel pathologic observations relevant to cognitive deficits in survivors of prematurity. Pediatr Res 2009;65:524-529.

21 Ball G, Boardman JP, Aljabar P, Pandit A, Arichi T, Merchant N, Rueckert D, Edwards AD, Counsell SJ: The influence of preterm birth on the developing thalamocortical connectome. Cortex 2013;49:1711-1721.

22 Tomassy GS, Berger DR, Chen HH, Kasthuri N, Hayworth KJ, Vercelli A, Seung HS, Lichtman JW, Arlotta P: Distinct profiles of myelin distribution along single axons of pyramidal neurons in the neocortex. Science 2014;344: 319-324.

23 Back S, Han B, Luo N, Chricton C, Xanthoudakis S, Tam J, Arvin K, Holtzman D: Selective vulnerability of late oligodendrocyte progenitors to hypoxia-ischemia. J Neurosci 2002;22:455-463

24 Jantzie LL, Cheung PY, Johnson ST, Bigam DL, Todd KG: Cerebral amino acid profiles after hypoxia-reoxygenation and $\mathrm{N}$-acetylcysteine treatment in the newborn piglet. Neonatology 2010;97:195-203.

25 Jantzie LL, Corbett CJ, Firl DJ, Robinson S: Postnatal erythropoietin mitigates impaired cerebral cortical development following subplate loss from prenatal hypoxia-ischemia. Cereb Cortex 2014, Epub ahead of print.

-26 Jantzie LL, Getsy PM, Firl DJ, Wilson CG, Miller RH, Robinson S: Erythropoietin atten- uates loss of potassium chloride co-transporters following prenatal brain injury. Mol Cell Neurosci 2014;61:152-162.

27 Jantzie LL, Talos DM, Jackson MC, Park HK Graham DA, Lechpammer M, Folkerth RD, Volpe JJ, Jensen FE: Developmental expression of N-methyl-D-aspartate (NMDA) receptor subunits in human white and gray matter: potential mechanism of increased vulnerability in the immature brain. Cereb Cortex 2013, Epub ahead of print.

28 Folkerth R, Haynes R, Borenstein N, Belliveau R, Trachtenberg F, Rosenberg P, Volpe J, Kinney $\mathrm{H}$ : Developmental lag in superoxide dismutases relative to other antioxidant enzymes in premyelinated human telencephalic white matter. J Neuropath Exp Neurol 2004;63:990999.

29 Smith AL, Alexander M, Rosenkrantz TS, Sadek ML, Fitch RH: Sex differences in behavioral outcome following neonatal hypoxia ischemia: insights from a clinical meta-analysis and a rodent model of induced hypoxic ischemic brain injury. Exp Neurol 2014;254:54-67.

30 Workman AD, Charvet CJ, Clancy B, Darlington RB, Finlay BL: Modeling transformations of neurodevelopmental sequences across mammalian species. J Neurosci 2013; 33:7368-7383.

- 31 Herlenius E, Lagercrantz H: Development of neurotransmitter systems during critical periods. Exp Neurol 2004;190:S8-S21.

32 Kelsom C, Lu W: Development and specification of GABAergic cortical interneurons. Cell Biosci 2013;3:19.

33 Kinney H, Back S: Human oligodendroglial development: relationship to periventricular leukomalacia. Semin Pediatr Neurol 1998;5: 180-189.

- $34 \mathrm{Xu} \mathrm{G}$, Broadbelt KG, Haynes RL, Folkerth RD, Borenstein NS, Belliveau RA, Trachtenberg FL, Volpe JJ, Kinney HC: Late development of the GABAergic system in the human cerebral cortex and white matter. J Neuropathol Exp Neurol 2011;70:841-858.

- 35 Robinson S, Petelenz K, Li Q, Cohen M, Buczek M, Lust D, Miller R: Developmental changes induced by prenatal hypoxia-ischemia insult in rats. Neurobiol Dis 2005; 18:568-581.

36 Back S, Luo N, Borenstein N, Levine J, Volpe J, Kinney H: Late oligodendrocyte progenitors coincide with the developmental window of vulnerability for human perinatal white matter injury. J Neurosci 2001;21:1302-1312.

37 Yang Y, Lewis R, Miller RH: Interactions between oligodendrocyte precursors control the onset of CNS myelination. Dev Biol 2011;350: 127-138.

38 Kuypers E, Ophelders D, Jellema RK, Kunzmann S, Gavilanes AW, Kramer BW: White matter injury following fetal inflammatory response syndrome induced by chorioamnionitis and fetal sepsis: lessons from experimental ovine models. Early Hum Dev 2012;88:931936. 
-39 Jantzie LL, Cheung PY, Obaid L, Emara M, Johnson ST, Bigam DL, Todd KG: Persistent neurochemical changes in neonatal piglets after hypoxia-ischemia and resuscitation with $100 \%, 21 \%$ or $18 \%$ oxygen. Resuscitation 2008;77:111-120.

-40 Eiby YA, Wright LL, Kalanjati VP, Miller SM, Bjorkman ST, Keates HL, Lumbers ER, Colditz PB, Lingwood BE: A pig model of the preterm neonate: anthropometric and physiological characteristics. PLoS One 2013;8:e68763.

-41 Rasch S, Sangild PT, Gregersen H, Schmidt M, Omari T, Lau C: The preterm piglet - a model in the study of oesophageal development in preterm neonates. Acta Paediatr 2010;99:201-208.

-42 Griffith JL, Shimony JS, Cousins SA, Rees SE, McCurnin DC, Inder TE, Neil JJ: MR imaging correlates of white-matter pathology in a preterm baboon model. Pediatr Res 2012;71: 185-191.

43 Dieni S, Inder T, Yoder B, Briscoe T, Camm E, Egan G, Denton D, Rees S: The pattern of cerebral injury in a primate model of preterm birth and neonatal intensive care. J Neuropath Exp Neurol 2004;63:1297-1309.

- 44 Delcour M, Russier M, Amin M, Baud O, Paban V, Barbe M, Coq J: Impact of prenatal ischemia on behavior, cognitive abilities and neuroanatomy in adult rats with white matter damage. Behav Brain Res 2012;232:233-244.

-45 Sab IM, Ferraz MM, Amaral TA, Resende AC, Ferraz MR, Matsuura C, Brunini TM, Mendes-Ribeiro AC: Prenatal hypoxia, habituation memory and oxidative stress. Pharmacol Biochem Behav 2013;107:24-28.

-46 Olivier P, Baud O, Evrard P, Gressens P, Verney C: Prenatal ischemia and white matter damage in rats. J Neuropathol Exp Neurol 2005;64:998-1006.

-47 Saadani-Makki F, Kannan S, Lu X, Janisse J, Dawe E, Edwin S, Romero R, Chugani D: Intrauterine administration of endotoxin leads to motor deficits in a rabbit model: a link between prenatal infection and cerebral palsy. Am J Obstet Gynecol 2008;199:651.e1-e7.

-48 Derrick M, Luo N, Bregman J, Jilling T, Ji X, Fisher K, Gladson C, Beardsley D, Murdoch G, Back S, Tan S: Preterm fetal hypoxia-ischemia causes hypertonia and motor deficits in the neonatal rabbit: a model for cerebral palsy. J Neurosci 2004;24:24-34.

-49 Kuypers E, Jellema RK, Ophelders DR, Dudink J, Nikiforou M, Wolfs TG, Nitsos I, Pillow JJ, Polglase GR, Kemp MW, Saito M, Newnham JP, Jobe AH, Kallapur SG, Kramer BW: Effects of intra-amniotic lipopolysaccharide and maternal betamethasone on brain inflammation in fetal sheep. PLoS One 2013; 8:e81644.

50 Cai Z, Pan Z-L, Pang Y, Evans O, Rhodes P: Cytokine induction in fetal rat brains and brain injury in neonatal rats after maternal lipopolysaccharide administration. Pediatr Res 2000;47:64-72.
51 Wang X, Rousset C, Hagberg H, Mallard C: Lipopolysaccharide-induced inflammation and perinatal brain injury. Semin Fetal Neonatal Med 2006;11:343-353.

52 Bergeron JD, Deslauriers J, Grignon S, Fortier LC, Lepage M, Stroh T, Poyart C, Sebire G: White matter injury and autistic-like behavior predominantly affecting male rat offspring exposed to group b streptococcal maternal inflammation. Dev Neurosci 2013;35: 504-515.

53 Uchida K, Nakahira K, Mimura K, Shimizu T, De Seta F, Wakimoto T, Kawai Y, Nomiyama M, Kuwano K, Guaschino S, Yanagihara I: Effects of Ureaplasma parvum lipoprotein multiple-banded antigen on pregnancy outcome in mice. J Reprod Immunol 2013;100:118127.

54 Jantzie LL, Corbett CJ, Berglass J, Firl DJ, Flores J, Mannix R, Robinson S: Complex pattern of interaction between in utero hypoxiaischemia and intra-amniotic inflammation disrupts brain development and motor function. J Neuroinflammation 2014;11:131.

55 Mazur M, Miller R, Robinson S: Postnatal erythropoietin treatment mitigates neural cell loss after systemic prenatal hypoxic-ischemic injury. J Neurosurg Pediatr 2010;6:206-221.

56 Dammann O, Leviton A: Intermittent or sustained systemic inflammation and the preterm brain. Pediatr Res 2014;75:376-380.

57 Cuenca AG, Wynn JL, Moldawer LL, Levy O: Role of innate immunity in neonatal infection. Am J Perinatol 2013;30:105-112.

58 Kotecha S, Mildner RJ, Prince LR, Vyas JR, Currie AE, Lawson RA, Whyte MK: The role of neutrophil apoptosis in the resolution of acute lung injury in newborn infants. Thorax 2003;58:961-967.

-59 Lin CY, Chang YC, Wang ST, Lee TY, Lin CF, Huang CC: Altered inflammatory responses in preterm children with cerebral palsy. Ann Neurol 2010;68:204-212.

-60 Hagberg H, Dammann O, Mallard C, Leviton A: Preconditioning and the developing brain. Semin Perinatol 2004;28:389-395.

61 Eklind S, Mallard C, Arvidsson P, Hagberg H: Lipopolysaccharide induces both a primary and a secondary phase of sensitization in the developing rat brain. Pediatr Res 2005;58: 112-116.

62 Yang L, Sameshima H, Ikeda T, Ikenoue T: Lipopolysaccharide administration enhances hypoxic-ischemic brain damage in newborn rats. J Obstet Gynaecol Res 2004;30:142-147.

63 van den Heuij LG, Mathai S, Davidson JO, Lear CA, Booth LC, Fraser M, Gunn AJ, Bennet L: Synergistic white matter protection with acute-on-chronic endotoxin and subsequent asphyxia in preterm fetal sheep. J Neuroinflammation 2014;11:89.
64 Van Steenwinckel J, Schang AL, Sigaut S, Chhor V, Degos V, Hagberg H, Baud O, Fleiss B, Gressens P: Brain damage of the preterm infant: new insights into the role of inflammation. Biochem Soc Trans 2014;42:557-563.

65 Gavilanes AW, Gantert M, Strackx E, Zimmermann LJ, Seeldrayers S, Vles JS, Kramer BW: Increased EEG delta frequency corresponds to chorioamnionitis-related brain injury. Front Biosci (Schol Ed) 2010;2:432-438.

66 Duncan JR, Cock ML, Loeliger M, Louey S, Harding R, Rees SM: Effects of exposure to chronic placental insufficiency on the postnatal brain and retina in sheep. J Neuropathol Exp Neurol 2004;63:1131-1143.

- 67 Rees S, Breen S, Loeliger M, McCrabb G, Harding R: Hypoxemia near mid-gestation has long-term effects on fetal brain development. J Neuropathol Exp Neurol 1999;58: 932-945.

68 Jellema RK, Lima Passos V, Zwanenburg A, Ophelders DR, De Munter S, Vanderlocht J, Germeraad WT, Kuypers E, Collins JJ, Cleutjens JP, Jennekens W, Gavilanes AW, Seehase M, Vles HJ, Steinbusch H, Andriessen $\mathrm{P}$, Wolfs TG, Kramer BW: Cerebral inflammation and mobilization of the peripheral immune system following global hypoxia-ischemia in preterm sheep. J Neuroinflammation 2013;10:13.

69 Jantzie LL, Miller RH, Robinson S: Erythropoietin signaling promotes oligodendrocyte development following prenatal systemic hypoxic-ischemic brain injury. Pediatr Res 2013;74:658-667.

-70 Segovia KN, McClure M, Moravec M, Luo NL, Wan Y, Gong X, Riddle A, Craig A, Struve J, Sherman LS, Back SA: Arrested oligodendrocyte lineage maturation in chronic perinatal white matter injury. Ann Neurol 2008;63: 520-530.

71 Drobyshevsky A, Jiang R, Derrick M, Luo K, Tan S: Functional correlates of central white matter maturation in perinatal period in rabbits. Exp Neurol 2014;261:76-86.

-72 Saadani-Makki F, Kannan S, Makki M, Muzik O, Janisse J, Romero R, Chugani D: Intrauterine endotoxin administration leads to white matter diffusivity changes in newborn rabbits. J Child Neurol 2009;24:1179-1189.

-73 Hickey E, Shi H, Van Arsdell G, Askalan R: Lipopolysaccharide-induced preconditioning against ischemic injury is associated with changes in toll-like receptor 4 expression in the rat developing brain. Pediatr Res 2011;70: $10-14$.

74 Robertson NJ, Tan S, Groenendaal F, van Bel F, Juul SE, Bennet L, Derrick M, Back SA, Valdez RC, Northington F, Gunn AJ, Mallard C: Which neuroprotective agents are ready for bench to bedside translation in the newborn infant? J Pediatr 2012;160:544-552. 